\title{
Les digues à paroi perforée, Caissons "JARLAN" de Dieppe : bilan général et expérience acquise
}

\author{
Bélorgey Michel \\ Professeur L.M.2.C. Groupe Mécanique des Fluides, Université de \\ Caen
}

\section{Résumé}

Le principe des digues à paroi perforée revient à Jarlan (1960) qui a proposé une alternative aux digues verticales afin de réduire le coefficient de réflexion. L'idée scientifique, basée sur la propagation des ondes acoustiques, était très originale. Cependant, durant les 20 ans qui ont suivi, les études entreprises sur ce type de structure n'ont essentiellement porté que sur la stabilité générale de l'ouvrage. En 1986 , le projet de construction de la nouvelle digue du port de Dieppe a donné l'occasion de définir un programme de recherche plus ambitieux, qui fut prolongé en 1996 dans le cadre du Programme Européen de Recherche "PROVERBS"

Les travaux que nous présentons ici sont le fruit de toute une équipe du laboratoire. Ils se rapportent à l'ensemble de ces deux programmes.

\section{Abstract}

The principle of the breakwaters with perforated wall is due to Jarlan (1960) who proposed an alternative to the vertical face breakwaters in order to reduce the coefficient of reflection. The scientific idea, based on the acoustic wave propagation, was very original. However, during the 20 years which followed, the studies undertaken on this type of structure primarily related only on the global stability of the breakwater. In 1986, the project of construction of the new breakwater of the port of Dieppe gave the opportunity to define a more ambitious program who was prolonged in 1996 within the framework of the European Research Program "PROVERBS ". This paper is the fruit of a whole team of the laboratory. They refer to the whole of these two programs.

\section{Introduction}

Les digues verticales sont couramment utilisées pour la protection des ports contre les vagues. Or ce type d'ouvrage a l'inconvénient de réfléchir la houle, ce qui entre autre accroît les difficultés de navigation au voisinage de ceux-ci. Depuis quelques années, des solutions sont apparues avec la réalisation d'ouvrages peu réfléchissants (types ARC, JARLAN, etc.). Mais leur fonctionnement hydraulique est encore mal connu., ce qui pénalise les possibilités d'optimisation.

L'origine de cette méconnaissance du fonctionnement hydraulique réside dans deux raisons principales :

* impossibilité pour les études sur modèles réduits, de respecter simultanément les conditions de similitude de Froude et de Reynolds. 
* absence d'une instrumentation non intrusive pour l'analyse du champ des vitesses au voisinage de la structure. En effet, de par son mouvement orbital, la houle engendre à l'aval d'une sonde matérielle un sillage qui suit le mouvement orbital et donc revient au point de mesure, perturbant ainsi les résultats.

La décision en 1986 de réaliser la nouvelle digue de Dieppe à l'aide de caissons "JARLAN" fut l'opportunité de la mise en place d'un programme de recherche plus ambitieux et spécifique à l'étude de ce type d'ouvrage.

Le programme associé à la digue de Dieppe était basé sur les principes suivants :

* associer pour un même ouvrage des études en laboratoire et des études in situ sur prototype de manière à pallier et quantifier les erreurs dues au non respect des règles de similitude, et contrôler la validation de la transposition des résultats du modèle réduit au cas réel.

* aborder l'étude à l'aide de l'analyse des efforts instantanés réels sur chacune des parois en fonction de la phase de la houle.

* exploiter les nouvelles techniques non intrusives de mesure des vitesses au sein d'un fluide (Vélocimétrie Laser) pour analyser le champ des vitesses généré par la houle au voisinage des structures (Bélorgey 1986) et ainsi pouvoir aborder l'étude du fonctionnement hydraulique des caissons "JARLAN".

Ce programme initial (1990), financé par le M.R.T. ( ${ }^{\circ}$ 90F0428) fut prolongé en 1996 dans le cadre du Programme Européen "PROVERBS" de MAST III (Probabilistic Design Tools for Vertical Breakwaters). Dans les deux cas, les travaux ont porté sur des études in situ et en laboratoire.

L'objectif était d'aboutir à une meilleure compréhension des phénomènes physiques, indispensable à une modélisation exploitable par les bureaux d'études. Les études ont porté sur:

- La dissipation d'énergie lors du passage de la houle à travers la paroi perforée.

- L'agitation à l'intérieur du caisson

- L'évolution du champ des vitesses et du champ turbulent au voisinage de la paroi perforée et dans le caisson.

- Les variations spatio-temporelles du champ des pressions sur les différentes faces du caisson, et les efforts associés

Les travaux que nous présentons ici sont le fruit des résultats de toute une équipe du laboratoire. Ils se rapportent à l'ensemble de ces deux programmes. Ils correspondent à l'analyse du bilan des résultats positifs, mais aussi des échecs qui sont également instructifs. Ils correspondent à :

- l'instrumentation de la digue, de Dieppe,

- l'étude relative à l'influence d'une plaque perforée seule,

- l'étude relative au caisson complet,

Ils ont conduit à l'aboutissement de 3 thèses et plusieurs rapports de DEA.

\section{Instrumentation de la digue de Dieppe.}

La nouvelle digue de Dieppe (225 $\mathrm{m}$ de long) qui prolonge l'ancienne digue ouest est orientée sud-nord. Elle est constituée de 9 doubles caissons JARLAN en béton armé ( 8 caissons et le musoir $-n^{\circ} 9$-) pour protéger des houles de S.W et N.E. Seul le caisson $n^{\circ} 6$ a été instrumenté (fig : 1). L'opération d'installation des capteurs a débuté en août 1991 par la pose des capteurs sous le radier lors de la construction 
des caissons dans la forme de radoub $\mathrm{n}^{\circ} 7$ du port du Havre. A cette date, 7 capteurs de pression Trans-America Instrument XI $1 \mathrm{~F}$ de $28 \mathrm{~mm}$ de diamètre ont été mis en place dans des cavités réalisées par carottage dans la base en béton. Le caisson concerné a alors été remorqué jusqu'à son site final de l'avant port de Dieppe. La deuxième phase du projet s'est déroulée durant l'année 1996. Elle a consisté en la pose de 8 capteurs identiques aux précédents, sur les parois de la chambre S.W. (houles les plus fortes) du caisson $n^{\circ} 6$, (Rousset, Bélorgey 1998). Les capteurs sont reliés par des câbles de $50 \mathrm{~m}$ à un système d'acquisition situé dans le bâtiment de la balise du musoir.

En mars 1998, un enregistreur de houle Inter Océan S4DW a été installé à $500 \mathrm{~m}$ au large de la digue de façon à coupler les données de houle aux mesures de pression.

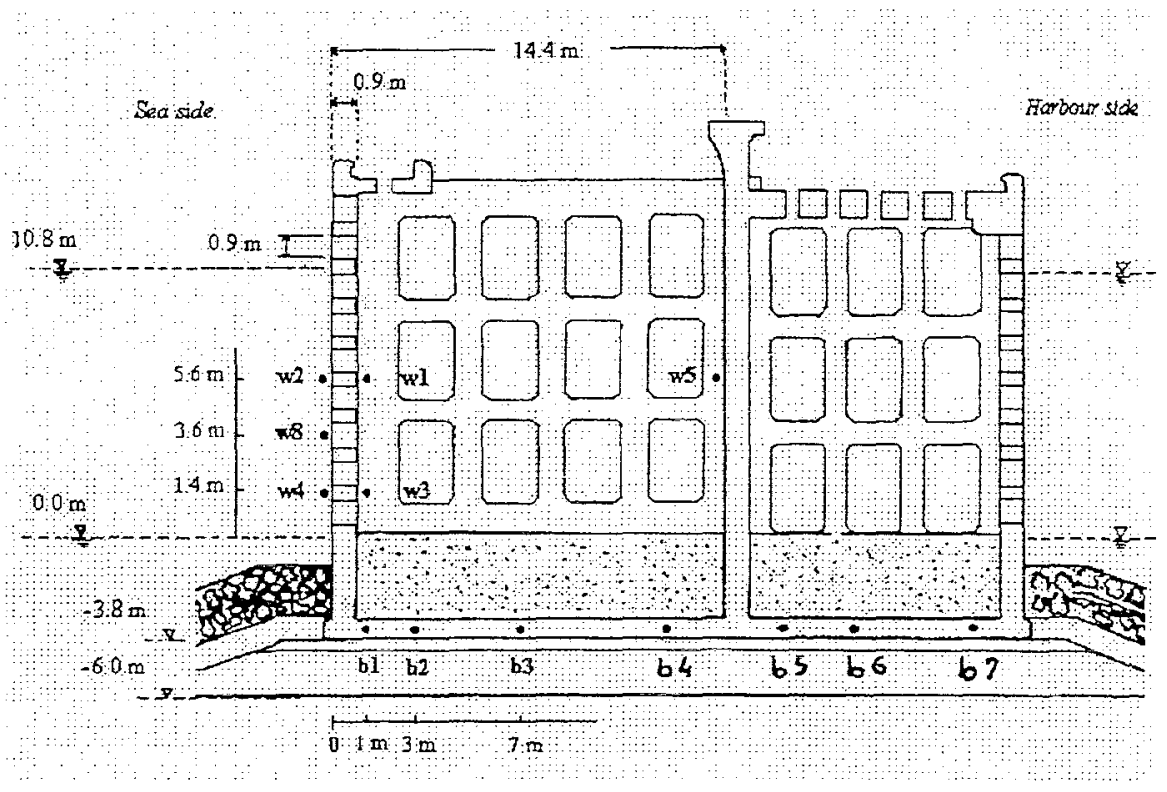

Fig : 1 Coupe du caisson $n^{\circ} 6$ de Dieppe, et position des capteurs de pression Cross section of Dieppe caisson and location of pressure cells

Des 7 capteurs placés sous le radier en 1991, 4 ne répondaient plus lors de l'exploitation des données $\left(b_{4}, b_{5}, b_{6}, b_{7}\right.$, $)$. Plusieurs causes peuvent être à l'origine de leur détérioration:

- l'installation du caisson lors de sa mise en place sur le site

- la tempête de février 1992 survenue alors que la digue n'était pas achevée et les câbles d'alimentation des capteurs non parfaitement protégés.

- le vieillissement en condition sévère entre le temps de pose 1991 et le temps d'exploitation (les premières mesures ont débuté en 1994)

\section{3. Étude du fonctionnement hydraulique.}

\subsection{Le modèle physique.}

Le modèle physique a été réalisé au $1 / 25$. Cette échelle a été choisie de manière à disposer d'un nombre de longueur d'onde suffisant entre le batteur et le modèle 
(pour un canal à houle de $24 \mathrm{~m}$ ). Elle a été prise aussi la plus grande possible pour limiter les effets d'échelle. Le diamètre des trous a cependant été choisi avec une légère distorsion de l'échelle $(40 \mathrm{~mm}$ au lieu de $36 \mathrm{~mm})$, de façon à augmenter le nombre de Reynolds. Ce choix renforce la turbulence, par contre la densité des trous à été choisie de façon à respecter la porosité du prototype $(28 \%)$.

Les essais sont exécutés dans le canal à houle du laboratoire $(24 \mathrm{~m}$ de long, $0.8 \mathrm{~m}$ de large, $1 \mathrm{~m}$ de profondeur). Le générateur de houle est constitué d'un batteur plan qui génère des houles régulières dont on peut faire varier la fréquence et l'amplitude.

Une série de sondes résistives permet l'enregistrement des variations du niveau de surface libre. Par ailleurs, le canal est équipé d'une chaîne de mesure des vitesses instantanées par vélocimétrie laser à deux composantes. Les essais ont été réalisés avec une hauteur d'eau de $\mathrm{h}=40 \mathrm{~cm}$ dans le canal, équivalente à une profondeur de $10 \mathrm{~m}$ en nature, proche des conditions réelles du site de Dieppe.

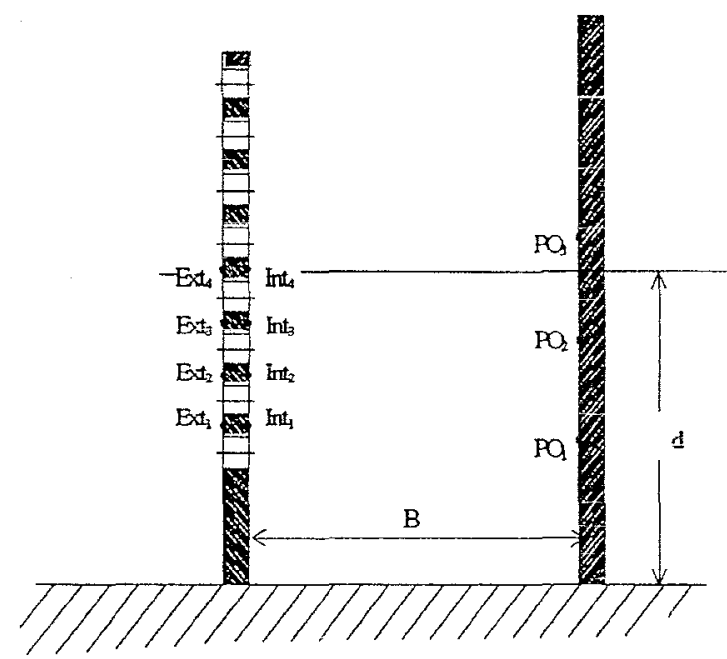

Fig 2a : Caisson sans berme Caisson without berm

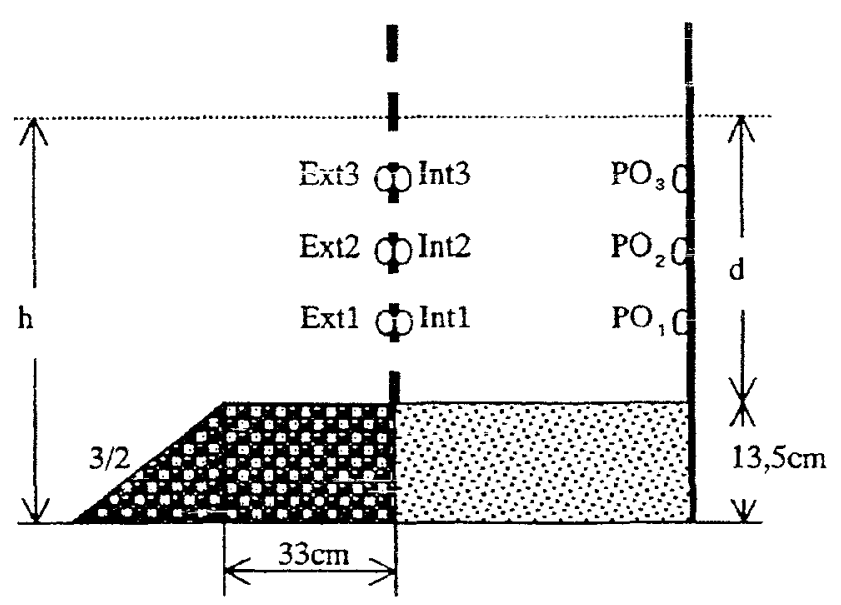

Fig $2 b$ : Caisson avec berme Caisson with berm

La mesure des efforts est obtenue par intégration du champ des pressions sur les diverses faces des parois du caisson. Pour cela nous avons disposé sur celles-ci un ensemble de mini capteurs Entran type FP 1142. Les figures $2 a$ et $2 b$ indiquent la position des capteurs dans les deux cas étudiés : caisson sans berme, posé directement sur le fond horizontal du canal, et caisson avec berme. Dans chacun des cas, les positions des capteurs par rapport au fond du canal, correspondaient à celles du prototype par rapport au zéro des cartes marines devant l'ouvrage.

\subsection{Fonctionnement hydraulique de la plaque perforée seule.}

Dans une première phase, pensant décomposer ce problème complexe en problèmes' plus simples nous avons étudié, en canal à houle, le cas de la plaque perforée seule avec une plage d'amortissement en fond de canal. Si les résultats obtenus présentent un intérêt scientifique, nous montrerons par la suite qu'ils ne peuvent en aucun cas apporter une aide à la compréhension des phénomènes physiques dans le cas du caisson complet. Bien au contraire. 


\subsubsection{Performances hydrauliques.}

L'étude à porté sur l'évolution des coefficients de réflexion $(\mathrm{Cr})$ et de transmission (Ct) de la houle en fonction de la cambrure de celle-ci, ainsi que sur la forme de la surface libre de part et d'autre de la plaque perforée. Les résultats obtenus pour une plaque de porosité de $28 \%$ sont indiqués sur la figures 3 pour différentes périodes de houles régulières $(1 \mathrm{~s} ; 1.4 \mathrm{~s} ; 1.8 \mathrm{~s} ; 2.2 \mathrm{~s}$ )

La principale conclusion de cette partie d'étude est la suivante : le comportement général de la structure est identique à un filtre et présente 3 modes en fonction de la cambrure de la houle $\gamma=\mathrm{H}_{\mathrm{i}} / \mathrm{L}$ :

$-\gamma<2 \%$, le filtre est pratiquement perméable.

$-2 \%<\gamma<7 \%$ le filtre est contrôlé par les effets de gravité dus à la différence de niveau d'eau de part et d'autre de la paroi.

$-7 \%<\gamma$, le filtre est saturé et les coefficients de réflexion et de transmission restent sensiblement constants.

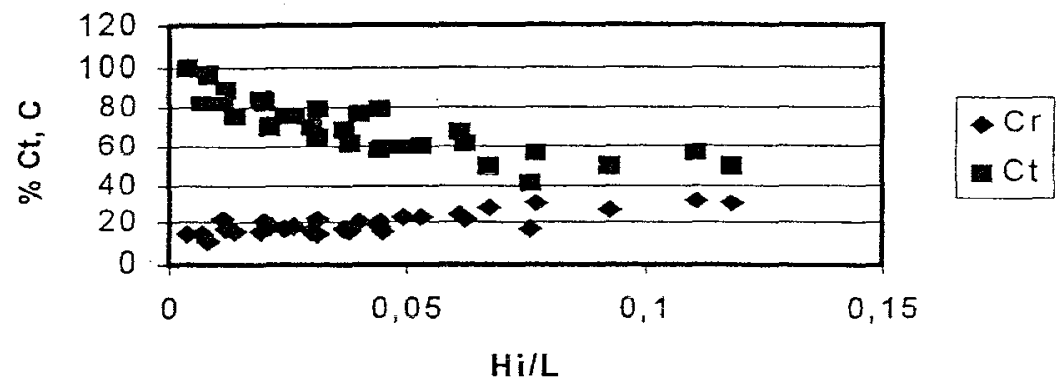

Fig 3 : Evolution de $\mathrm{Cr}$ et $\mathrm{Ct}$ en fonction de la cambrure de la houle Evolution of $\mathrm{Cr}$ and $\mathrm{Ct}$ versus incident wave steepness

\subsubsection{Champ des vitesses et champ turbulent}

L'analyse du champ des vitesses et du champ turbulent ont été réalisés de part et d'autre de la paroi perforée, pour différents paramètres de houle et en fonction de la phase de celle-ci. Fig $4 a$ et $4 b$.

Les résultats obtenus montrent:

- une répartition sensiblement uniforme à l'aval des orifices

- une vitesse sensiblement identique pour chaque orifice

- une énergie turbulente sensiblement identique pour chaque orifice (l'énergie turbulente $u^{\prime 2}+\nabla^{\prime 2}$ étant représentée sur la figure par le diamètre d'un cercle)

Par ailleurs, ils montrent aussi qu'à environ 3 diamètres des trous la houle se reforme sans perturbation.

\subsection{Fonctionnement hydraulique du caisson avec paroi perforée}

La deuxième phase de l'étude en canal a porté sur le caisson complet. Les analyses ont été réalisées pour différents paramètres de houle (Hauteur incidente Hi et longueur d'onde $\mathrm{L}$ ) en fonction de la largeur relative de la chambre $\mathrm{B} / \mathrm{L}$. Nos résultats mettent en évidence : 
a) Une évolution quasi "parabolique" du coefficient de réflexion $\mathrm{Cr}$ en fonction de $\mathrm{B} / \mathrm{L}$ présentant un minimum pour une valeur de $\mathrm{B} / \mathrm{L}$ d'environ 0.18 . (Fig 5 ). Ces résultats confirment ceux de Suh et Park (1995).

b) L'influence de la berme et du lest sur la valeur minimum de $\mathrm{Cr}$. (Fig 5).

c) Une surélévation du niveau moyen de la surface libre à l'intérieur du caisson présentant un maximum pour une valeur de $\mathrm{B} / \mathrm{L}$ analogue à celle de $\mathrm{Cr}$ minimum. (Fig 6)

d) Une répartition des vitesses au voisinage des orifices (Fig 7) très différente de celle correspondant à la plaque seule (Fig 4a).

- le champ des vitesses est très différent selon la hauteur de l'orifice

- les vitesses à la sortie de l'orifice ne sont plus uniformes, seule une partie de l'orifice participe à l'écoulement. Ce résultat remet en cause les modèles de calcul des pertes de charge classiquement utilisés pour ce type de problème et montre l'influence du mouvement de la masse fluide au sein de la chambre.

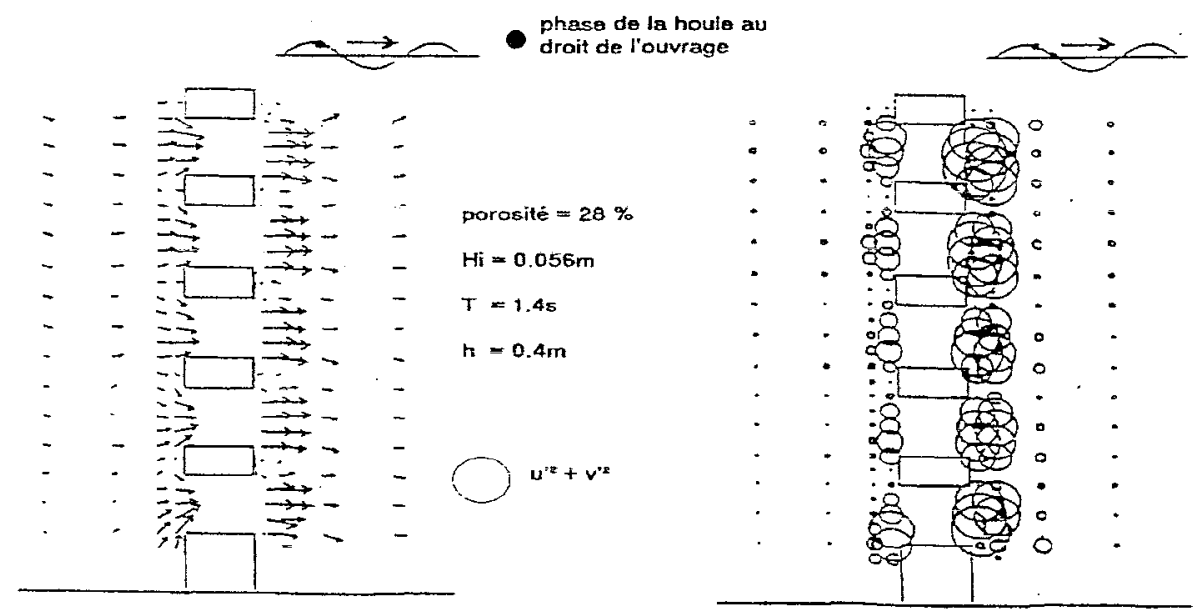

Fig 4 : Fonctionnement d'une paroi perforée seule

a : Exemple de champ des vitesses

Example of velocity field b : Exemple de champ turbulent Example of turbulent field

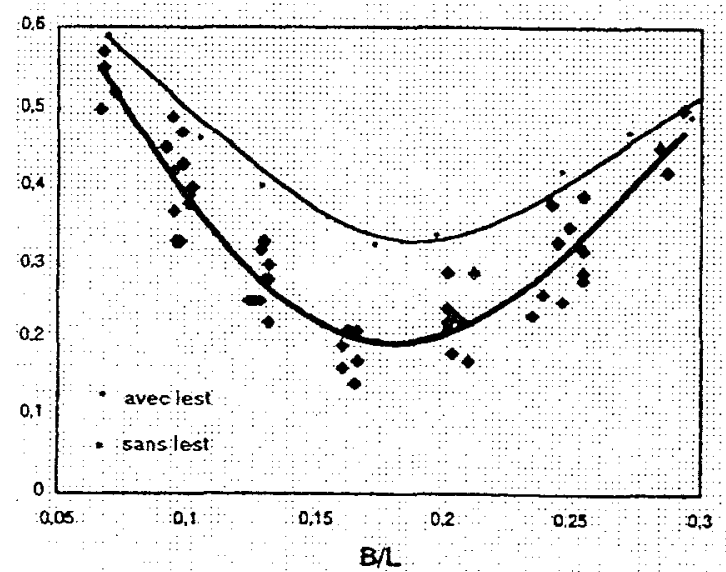

Fig 5 : Evolution de $\mathrm{Cr}$

Evolution of $\mathrm{Cr}$

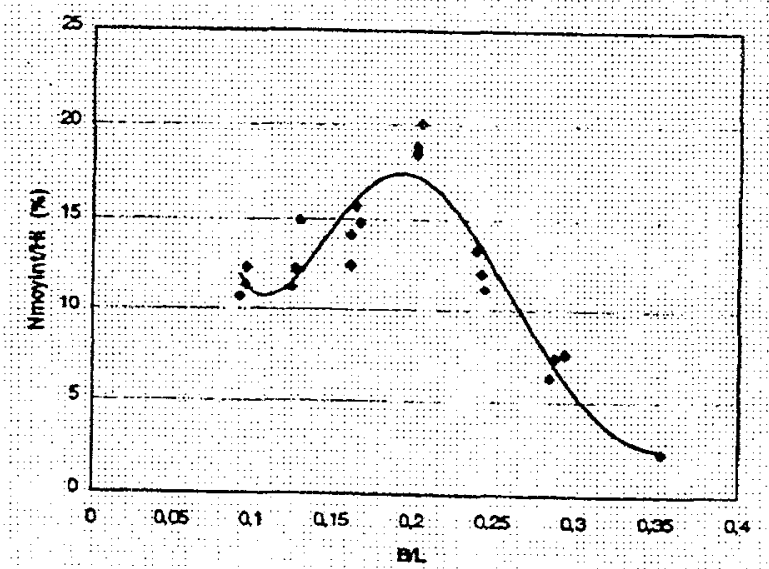

Fig 6 : Evolution du niveau moyen relatif Nmoyen/Hidans la chambre Evolution of mean wate level in chamber 
Par ailleurs, le champ des vitesses au sein du caisson complet présente des fluctuations importantes, et moyenné sur une période, une circulation générale qui dépendent du rapport B/L. (Fig 8). Par contre, il faut souligner que dans ce cas, une première analyse réalisée avec un pas de mesure trop grand $(2 \mathrm{~cm})$ conduisait à des résultats aberrants, et que pour pouvoir conclure plus nettement sur ce point il conviendrait de réduire le pas (ici de $1 \mathrm{~cm}$ ) à une valeur encore plus faible (en dessous de $0.5 \mathrm{~cm}$ ).

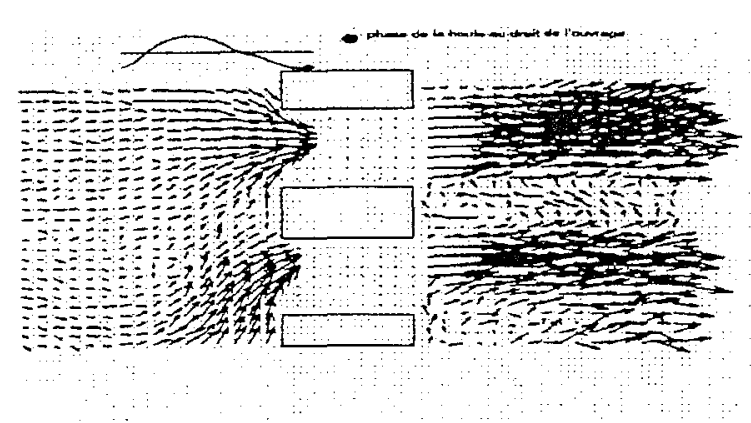

Fig 7 : Exemple de champ des vitesses au voisinage d'orifice de la paroi du caisson Example of velocity field near the orifice of the perforated wall of the caisson

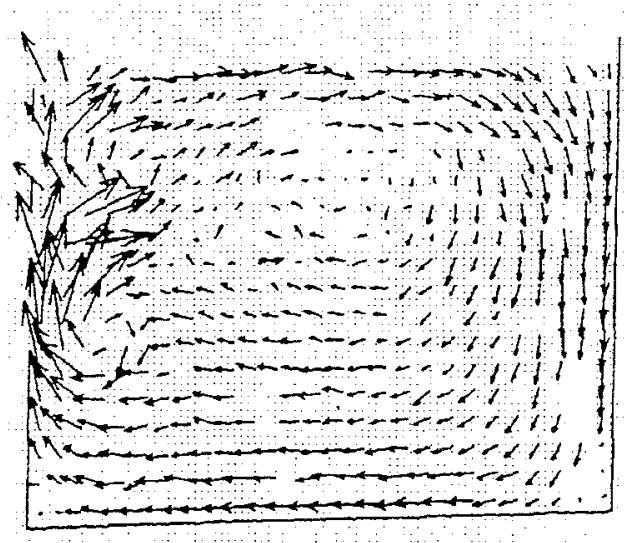

Fig 8: Exemple de champ des vitesses dans le caisson

Example of velocity field inside the wave chamber

\section{Analyse des pressions et des efforts.}

\subsection{Analyse des efforts sur les parois}

Les études, tant sur le prototype de Dieppe que sur le caisson en canal ont portées sur la mesure des pressions instantanées sur chacune des parois du caisson, de manière à connaître, par intégration des champs de pression, les efforts instantañés sur chacune des parois en fonction de la phase de la houle. Nous avons donc analysé séparément les efforts sur:

- la face extérieure de la paroi perforée : Fext.

- la face intérieure de la paroi perforée : Fint.

- la paroi opaque: Fr.

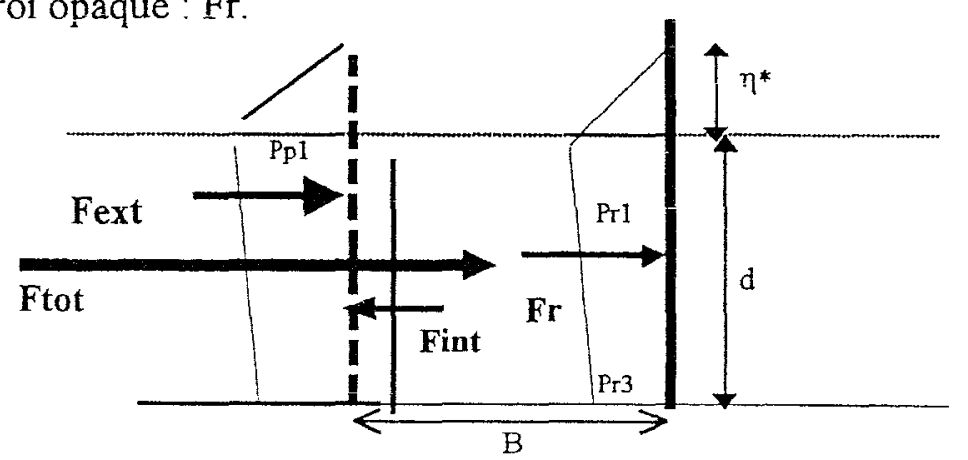

Fig 9 : Diagramme des pression et efforts résultants Pressure diagrams and resultani forces 


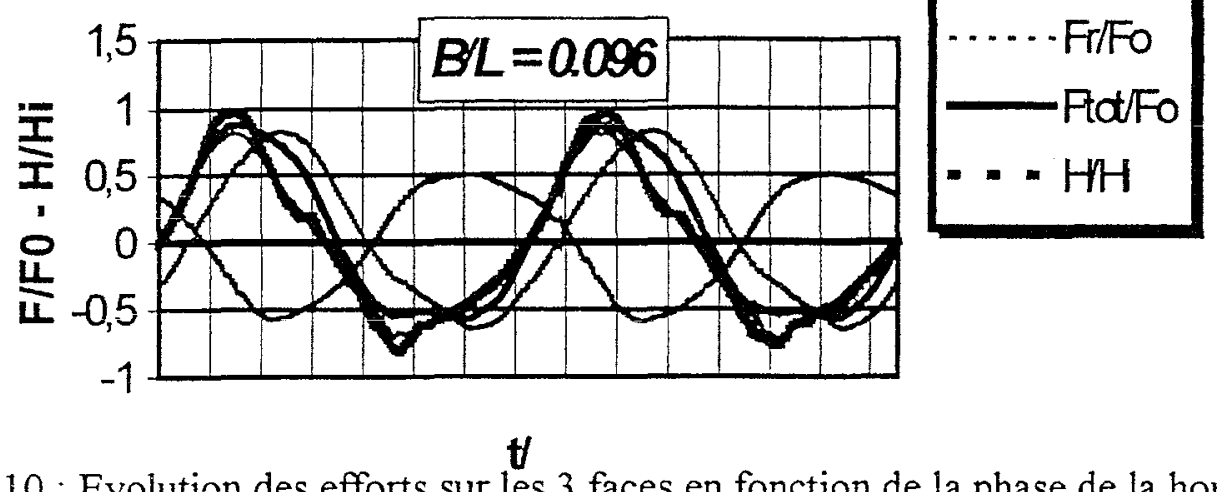

Fig 10 : Evolution des efforts sur les 3 faces en fonction de la phase de la houle Time histories of forces at the 3 faces of the breakwater

Et réálisé la somme des valeurs en fonction de la phase de la houle (Fig 9).

La figure 10 donne un exemple d'évolution des efforts instantanés sur chacune des faces du caisson en fonction de la phase de la houle. On peut remarquer:

- le déphasage entre les efforts sur chacune des parois et ceiui de la résultante par rapport à la phase de la houle au niveau de la paroi perforée.

- une poussée sur la paroi perforée qui peut devenir négative en fonction de la phase de la houle.

Pour le calcul des efforts (Bélorgey \& al 1999) nous avons exploité la formule de Goda (1985) modifiée par Takahashi et al (1994)

De ces résultats, nous déduisons:

- la force résultante sur la paroi perforée Fp, égale à la somme des poussées sur les faces externe et interne. ( $\mathrm{Fp}=\mathrm{Fext}+\mathrm{Fint})$

- la force sur la paroi opaque Fr

- la résultante générale des poussées sur le caisson, égale à la somme des poussées sur chacune des parois.

La comparaison entre la résultante réelle, des efforts calculés par intégration des valeurs instantanées des pressions sur les 3 parois (Fig 10) et celle calculée à l'aide de la formulation de Goda-Takahashi, nous a conduit à introduire un coefficient correcteur $\chi$ permettant de tenir compte des conditions de simultanéité des efforts sur les parois. Ce coefficient correcteur $\chi$ est fonction du rapport $\mathrm{B} / \mathrm{L}$.

La résultante des forces s'exprime alors sous la forme :

$$
\text { Ftot }=\chi(\mathbf{F p}+\mathbf{F r})
$$

\subsection{Formulation analytique du coefficient correcteur $\chi$}

Les réflexions qui ont conduit à la formulation du coefficient $\chi$ sont les suivantes :

$-\mathrm{B} / \mathrm{L}=0 \Rightarrow \chi=1$, (la structure doit se comporter comme une paroi opaque).

- $\mathrm{B} / \mathrm{L} \rightarrow \infty \Rightarrow \chi=1$, (les deux parois deviennent indépendantes car comme nous l'avons vu, la houle se reforme à l'arrière de la paroi perforée isolée). 


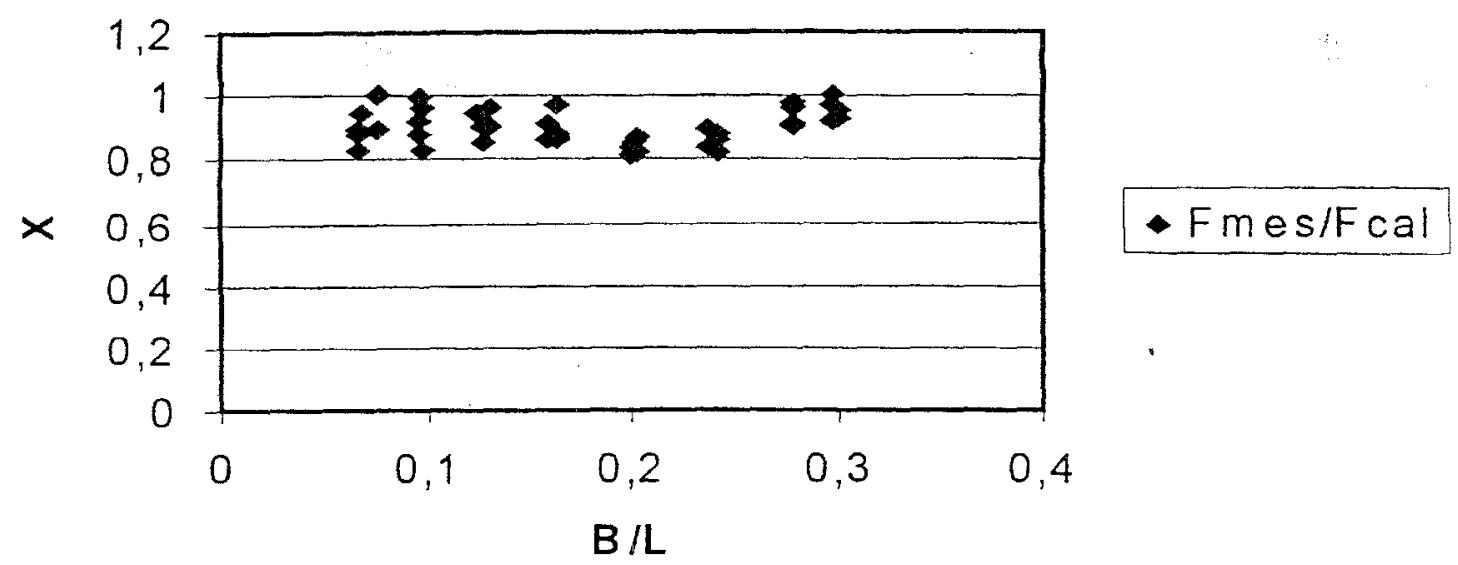

Fig 11 : Evolution de $\chi$ en fonction de $B / L$ Evolution of $\chi$ versus $B / L$

La figure 11 représente l'évolution du coefficient $\chi$ en fonction de la largeur relative de la chambre $(B / L)$

- Pour $\mathrm{B} / \mathrm{L}$ voisin de $0.2, \chi$ présente un minimum. Or c'est pour cette valeur que l'énergie dissipée est maximale ( $\mathrm{Cr}$ minimum et niveau moyen de Surface Libre maximum).

Une telle évolution prenant en compte:

- les conditions aux limites citées plus haut,

- la présence d'une valeur extrême (minimale ici)

est analogue à celle que nous avons trouvée dans l'étude des tensions de Reynolds au sein d'une couche limite générée par la houle sur les fonds marins, (ce qui nous permet de supposer que de la turbulence au sein du caisson n'est pas sans influence sur le phénomène).

Les fonctions analytiques d'une variable $\mathrm{x}$ qui représentent le mieux ce type d'évolution s'écrivent sous la forme :

$$
f(x)=g(x) \cdot e^{-h(x)}
$$

Aussi, par analogie, en prenant en compte les conditions aux limites et en faisant l'hypothèse au vu des résultats, que la tangente à l'origine est horizontale, nous proposons d'exprimer le coefficient correcteur $\chi$ sous la forme :

$$
\chi=1-a \cdot(B / L)^{2} \cdot e^{-(B / L) / b}
$$

Où $\mathrm{a}$ et $\mathrm{b}$ sont des constantes qui dépendent des paramètres de construction des caissons (forme des trous, épaisseur de la paroi perforée, nature de la berme etc.) et éventuellement des conditions hydrauliques.

Dans le cas précis de notre étude et pour les paramètres qui sont les nôtres :

$$
a=15.24 \quad \text { et } \quad b=0.1
$$


Un prochain programme de recherche devrait nous permettre de prolonger cette analyse de façon à quantifier l'influence des divers paramètres de design du caisson sur la valeur de ces coefficients.

\section{Remerciements.}

Les études ont été réalisées grâce au support du M.R.T. (contrat 90 F 0428) et de la C.E. (Programme Européen MAST III "PROVERBS")

\section{Equipe du laboratoire ayant participé au programme.}

Bélorgey M. ; Vasselin E. ; Rousset J.M. ; Caminade D. ; Lambert E. ; Tabet Aoul Khadir A. ; Zouatine M. ; Szklarek Ph. ; Arsié A. ;

\section{Références bibliographiques.}

Bélorgey M. Le Bas J., Grandjean A. Application of Laser Doppler Velocimetry to the study of turbulence generated by swell in the vicinity of walls or obstacles. Coastal Engineering, vol.13, pp. 183-203, 1989.

Bélorgey M., Khadir A., Tabet-Aoul, Vasselin E._Etude en similitude des digues peu réfléchissantes. Journées Génie Civil. Nantes 1995

Bélorgey $\mathrm{M}$. Bergman $\mathrm{H}$. de Gerloni M., Rousset J.M., Tabet Aoul. Perforated Caisson Breakwaters : Wave loads and hydraulic performance. Coastal Stuctures 99 Santander, Espagne, June, 1999

Caminade D. Interaction houle-structures. Etude de la transmission de la houle à travers une paroi perforée (type Jarlan). Thèse de Doctorat (Le Havre) 1992

De Gerloni M., Bélorgey M., Bergman H., Colombo D., Franco L., Passoni G., Rousset J.M., Tabet-Aoul, Alternative low reflection structures : perforeted vertical walls. Chap. 8 du rapport final PROVERBS (MAST III) 33 pages Février 1999

Goda Y. Random seas and Design of Maritime Structures. University of Tokyo Press. 1985

Rousset J.M. Bélorgey M. Analyse des variations de pressions générées par la houle sur la digue Jarlan du port de Dieppe. 5ièmes Journées Génie Civil - Génie Côtier, Toulon, mai 1998

Rousset J.M. Tabet Aoul, Bélorgey M. Analysis of Horizontal Forces on the Vertical Walls of Perforated Breakwater. $9^{e}$ International Offshore and Polar Engineering Conference (ISOPE). Brest, France, May 30-June 4 - 1999

Rousset J.M. Kohl D. Bélorgey M. Phénomènes dissipatifs dans une digue à paroi perforée. VT ièmes Journées Génie Civil Génie Côtier, Caen 2000

Rousset. Sollicitation d'une digue Jarlan sous l'action de houles aléatoires. Thèse de Doctorat (Université de Caen) 2000

Tabet Aoul, Bélorgey M. Etude de la circulation à l'intérieur d'un caisson Jarlan. Toulon Vièmes Journées Génie Civil - Génie Côtier, Toulon 1998

Tabet-Aoul. Etude expérimentale du fonctionnement Hydraulique des digues à parois perforées. Thèse de Doctorat (Université du Havre) 1998

Takahashi S., Shimosako K. Wave pressure on a perforated wall. Proceeding of Intern. Conf. On Hydro-technical Eng. for Port and Harbor. (1994) 